\title{
What the online manipulation of linguistic activity can tell us about language and thought
}

\author{
Lynn K. Perry* and Gary Lupyan \\ Department of Psychology, University of Wisconsin-Madison, Madison, WI, USA \\ *Correspondence: Ikperry@wisc.edu \\ Edited by: \\ Leonid Perlovsky, Harvard University and Air Force Research Laboratory, USA
}

Keywords: verbal interference, transcranial direct current stimulation (tDCS), language and thought, linguistic relativity, labeling

Questions about the relationship between language and thought have long fascinated psychologists, philosophers, and the general public. One specific question is the extent to which verbal labels causally impact cognitive processes-how does calling an object by a particular name influence the way people categorize it; how does knowing words for mental states influence our reasoning about the minds of others; how does learning and using words like left influence our navigation behavior? One way to learn how the words we use to label objects, mental states, or locations affect our thoughts is to increase or decrease the ease with which we can use these words and observe outcomes of these manipulations on "non-linguistic" tasks. For example, if the word left enables us to remember which way to turn, preventing its activation might be expected to disrupt navigation. Manipulating the labeling process (and the engagement of language more broadly) is therefore very useful in exploring how language influences cognition. In this paper, we review two methodologies for implementing linguistic manipulations: verbal interference and transcranial direct current stimulation (tDCS), and discuss what we can learn about the role of language in cognitive processes from this line of research.

\section{VERBAL INTERFERENCE}

The primary method for manipulating linguistic activity is the use of verbal interference. The logic of verbal interference is well summarized by Winawer et al. (2007) in the context of studying the effects of language on color perception:

"[I]f linguistic processes play an active, online role in perceptual tasks, then a verbal dual task, but not a non-linguistic dual task, should diminish the goluboy/siniy [light blue / dark blue] category advantage found in Russian speakers," (Winawer et al., 2007, p. 7781).

Winawer et al. reported that Russian speakers appear to perceive a larger perceptual distinction between light and dark blues, consistent with a lexical difference between these categories in Russian. One possibility is that these differences stem from long-term perceptual learning caused by years of distinguishing colors in one language (e.g., Özgen and Davies, 2002). An alternative is that the cross-linguistic perceptual differences arise from online top-down influences of language (e.g., Fonteneau and Davidoff, 2007; Lupyan, 2008). If true, then disrupting these top-down effects in some way may disrupt these online effects of language eliminating the cross-linguistic difference-the pattern observed in the study. It is important to note that for verbal interference to have some effect does not require the involvement of language to be strategic. Rather, the involvement could be one of "the spontaneous but unspoken use of lexical codes," (Gilbert et al., 2006, p. 489). On this account, previous associations of using the word blue to describe the color blue cause the word to become reactivated when the color is seen (Lupyan, 2012a,b). The automatic recruitment of color labels may temporarily warp the perceptual space, producing cross-linguistic differences of the sort observed by Winawer et al.

Using similar logic, verbal interference has been used to argue for a role of language in number concepts (Frank and Barner, 2012), spatial memory (HermerVazquez et al., 1999), categorization
(Lupyan, 2009), and theory of mind (Newton and de Villiers, 2007). A similar logic underlies behavioral up-regulation of linguistic processes through redundant presentation of labels (e.g., Lupyan et al., 2007) or overt self-directed speech (Lupyan and Swingley, 2012). If internally generated labels support some cognitive or perceptual process, then redundant externally-presented labels can be thought to up-regulate the linguistic contribution and verbal interference to down-regulate it.

Verbal interference has also been used to examine mechanisms of developmental change. For example, in a task requiring participants to locate an object hidden in the corner of a room, children rely on the room's shape, while adults use one of the walls-painted in a distinct color from the other walls- to find the object (Hermer and Spelke, 1994; cf. Twyman and Newcombe, 2010). While developments in spatial language correlate with developments in using the wall as a landmark, this cannot tell us whether language causally influences spatial memory. In order to determine whether language supports development of spatial memory, Hermer-Vazquez et al. (1999) used a verbal interference paradigm. When adults performed the task while shadowing speech, their performance mirrored that of children's. Thus, in addition to improving our understanding of how language affects cognition in real-time, verbal interference has improved our understanding of how influences of language on cognition develop.

However, the use of verbal interference is not without problems. First, despite being used for many years across many domains, there is at present no working theory of verbal interference. Put 
bluntly: no one is sure how it works. Its use is vaguely based on the notion that language is a unitary system that can be disrupted, but this assumption is potentially problematic given the degree to which language-related activity is distributed both anatomically (JungBeeman, 2005) and functionally, e.g., syntax and semantics, rather than being entirely dissociable systems, tightly interact (MacDonald et al., 1994). Additionally, there is little agreement as to what constitutes verbal interference. Researchers have used numerous tasks, with little theoretical basis for choosing one over another. Tasks falling under the umbrella of verbal interference have included: rehearsing multi-digit numbers for later memory tests (e.g., Gilbert et al., 2006; Lupyan, 2009), repeating the letters “a,b,c" (e.g., Emerson and Miyake, 2003); alternating between naming months and days (e.g., Baddeley et al., 2001), making rhyme judgments (Roberson et al., 2007), answering factual questions such as "What is your name?" (e.g., Hatano et al., 1977), and repeating text, known as speech shadowing (Hermer-Vazquez et al., 1999; Frank and Barner, 2012). These interference tasks differ both in general difficulty and the ease with which performance can be assessed. For example, an interference task with a memory component gives an indication of how well participants rehearsed information-a proxy for how much effort was put into the verbal task-repetition of "a,b,c" does not. Such inconsistencies makes it hard (1) to infer why verbal interference sometimes interferes with primary task performance and sometimes does not and (2) to assess what aspect of language is recruited in the primary task which, when interfered with, disrupts performance.

A final problem with verbal interference is that it requires participants to perform two tasks simultaneously. It is therefore necessary to use a control interference task to determine which changes in primary task performance stem from manipulation to specifically linguistic processes and which stem from having to perform two tasks. Control tasks also vary widely across experiments: from tests of visuospatial memory (e.g., Gilbert et al., 2006; Lupyan, 2009) to foot tapping (Baddeley et al., 2001; Emerson and Miyake, 2003), and rhythm-shadowing (Hermer-Vazquez et al., 1999). Importantly, unless interference tasks are equated in all ways except for their "verbality," little can be said about the role of language in the primary task. For example, it has been argued that verbal, but not non-verbal interference disrupted performance on a falsebelief task (Newton and de Villiers, 2007). However, when the interference tasks were better equated for difficulty, both were similarly disruptive (Dungan and Saxe, 2012). Additionally, because verbal interference uses a dual-task paradigm, participants can exert different amounts of effort into the tasks. Such differential effort is difficult both to measure and control.

Despite its appeal, verbal interference paradigms have clear shortcomings. Below, we outline an alternative way of perturbing linguistic processes that solve some of the shortcomings, and advocate for systematic cross-method comparisons of linguistic perturbation methods to more fully inform our understanding of how language augments cognition and perception.

\section{TRANSCRANIAL DIRECT CURRENT STIMULATION}

One way to avoid some challenges posed by verbal interferences is by manipulating language processing without using secondary tasks through the use of noninvasive brain stimulation. Here, we focus on one such method-tDCS a painless method of regulating cortical excitability through weak electrical current to the scalp-that allows the experimenter to subtly up- and down-regulate neural activity over cortical areas implicated in language processing. For example, using tDCS to up-regulate activity over Wernicke's area (associated with aspects of labeling, particularly comprehension of word meaning; e.g., Price, 2000) is associated with increased ability to map novel words to pictures (Flöel et al., 2008) using it to up-regulate activity over Broca's area (associated with linguistic processes such as speech production; e.g., Gernsbacher and Kaschak, 2003) is associated with increased artificial grammar learning (de Vries et al., 2010).

Similar to using verbal interference, using tDCS to study linguistic influences on cognition assumes that language is a system that can be selectively perturbed. However, tDCS avoids the need to use dual task paradigms. The participant simply performs the main task while undergoing tDCS which, depending on the electrode arrangement, either up- or down-regulates cortical activity in targeted regions. Up-regulating activity is theoretically analogous to behavioral up-regulation through presentation of overt labels (Lupyan, 2008) or behavioral self-directed speech (Lupyan and Swingley, 2012); down-regulating activity is theoretically analogous to verbal interference.

Recently, Lupyan et al. (2012) examined effects of tDCS on non-verbal categorization, showing that down-regulating activity over Broca's area was associated with impairments in the ability to form categories that required selectively representing specific perceptual features to the exclusion of others, e.g., GREEN THINGS, a deficit similar to one shown by individuals performing verbal interference (Lupyan, 2009) or by those with language impairments such as aphasia (Davidoff and Roberson, 2004; Lupyan and Mirman, 2013). In avoiding pitfalls of a dual task design, however, Lupyan and colleagues' tDCS study allows for more definitive conclusions about mechanisms by which language might affect categorization because participants all completed the same task, and between-group differences can be linked to changes in neural activity in a particular cortical region providing at least a foothold for starting to connect behavioral data on the role of language in categorization to particular neural mechanisms.

\section{IMPORTANT FUTURE CONSIDERATIONS}

Although tDCS may be well-suited for manipulating linguistic activity, at present there are no direct comparisons of tDCS to verbal interference. It would be useful to know whether domains in which we have seen effects of verbal interference on performance are affected by tDCS over areas associated with language processes and whether domains in which we have not previously seen effects are similarly unaffected. For example, tDCS can 
be used to determine if language-related cortical regions shown to be recruited in non-verbal color-judgment tasks (e.g., Ting Siok et al., 2009) are causally implicated by showing perturbations with tDCS have behavioral consequences in colorjudgment, thereby informing the neural basis of language-augmented perception. tDCS also has the potential to open up additional domains to investigate effects of language on cognition without the errorfraught hunt for perfect control interference tasks.

In sum, we advocate: (1) a more systematic comparison of linguistic perturbation methods, specifically comparing behavioral linguistic perturbations to perturbations utilizing stimulation techniques such as tDCS; (2) a more rigorous comparison of different verbal interference tasks; and (3) a theory that elucidates the mechanisms by which verbal interference actually works. Such an examination will be critical to clarifying the contributions of language to cognition, helping us answer such questions as whether the mechanisms by which language affects perception of color categories are in some broad sense similar to mechanisms by which language affects spatial cognition. Performing the cross-method comparisons we advocate will highlight possible contradictions leading to further theoretical refinement. For example, what would it mean for the underlying cognitive and neural processes if one verbal interference method affects a primary task but another does not?

The extant empirical literature on effects of language on cognition and perception takes us considerably beyond the question as it is often phrased: "Does language affect thought," (Boroditsky, 2010a). This literature (e.g., Gentner and Goldin-Meadow, 2003; Casasanto, 2008; Boroditsky, 2010b; Lupyan, 2012a,b), while rich in demonstrations, requires a more rigorous investigation of the mechanisms by which learning and using language augment and perhaps fundamentally alter cognition and perception. We believe significant clarity on this important question can be achieved by combining creative uses of linguistic perturbation techniques with theoretical refinement of their mechanisms.

\section{ACKNOWLEDGMENTS}

We would like to thank Pierce Edmiston for his comments on an earlier version of this paper.

\section{REFERENCES}

Baddeley, A., Chincotta, D., and Adlam, A. (2001). Working memory and the control of action: evidence from task switching. J. Exp. Psychol. Gen. 130, 641-657. doi: 10.1037/0096-3445.130. 4.641

Boroditsky, L. (2010a). Do the languages we speak shape the way we think? in The Economist. Available online at: http://www.economist.com/ debate/overview/190

Boroditsky, L. (2010b). "How the languages we speak shape the ways we think: the FAQs," in The Cambridge Handbook of Psycholinguistics, eds M. J. Spivey, M. Joanisse, and K. McRae (Cambridge: Cambridge University Press), 615-632.

Casasanto, D. (2008). Who's afraid of the big bad whorf? Crosslinguistic differences in temporal language and thought. Lang. Learn. 58, 63-79. doi: 10.1111/j.1467-9922.2008.00462.x

Davidoff, J., and Roberson, D. (2004). Preserved thematic and impaired taxonomic categorisation: a case study. Lang. Cogn. Processes 19, 137-174. doi: 10.1080/01690960344000125

de Vries, M. H., Barth, A. C. R., Maiworm, S., Knecht, S., Zwitserlood, P., and Flöel, A. (2010). Electrical stimulation of Broca's area enhances implicit learning of an artificial grammar. J. Cogn. Neurosci. 22, 2427-2436. doi: 10.1162/jocn.2009. 21385

Dungan, J., and Saxe, R. (2012). Matched falsebelief performance during verbal and nonverbal interference. Cogn. Sci. 36, 1148-1156. doi: 10.1111/j.1551-6709.2012.01248.x

Emerson, M. J., and Miyake, A. (2003). The role of inner speech in task switching: a dual-task investigation. J. Mem. Lang. 48, 148-168. doi: 10.1016/S0749-596X(02)00511-9

Flöel, A., Rösser, N., Michka, O., Knecht, S., and Breitenstein, C. (2008). Noninvasive brain stimulation improves language learning. J. Cogn. Neurosci. 20, 1415-1422. doi: 10.1162/jocn.2008.20098

Fonteneau, E., and Davidoff, J. (2007). Neural correlates of colour categories. Neuroreport 18 , 1323-1327. doi: 10.1097/WNR.0b013e3282c48c33

Frank, M. C., and Barner, D. (2012). Representing exact number visually using mental abacus. J. Exp. Psychol. Gen. 141, 134-149. doi: 10.1037/ a0024427

Gentner, D., and Goldin-Meadow, S. (2003) Language in Mind: Advances in the Study of Language and Thought. Cambridge, MA: MIT Press.

Gernsbacher, M. A., and Kaschak, M. P. (2003). Neuroimaging studies of language production and comprehension. Annu. Rev. Psychol. 54, 91-114. doi: 10.1146/annurev.psych.54.101601. 145128

Gilbert, A. L., Regier, T., Kay, P., and Ivry, R. B. (2006). Whorf hypothesis is supported in the right visual field but not the left. Proc. Natl. Acad. Sci. U.S.A. 103, 489-494. doi: 10.1073/pnas.050986 8103
Hatano, G., Miyake, Y., and Binks, M. G. (1977). Performance of expert abacus operators. Cognition 5, 47-55. doi: 10.1016/0010-0277 (77)90016-6

Hermer, L., and Spelke, E. S. (1994). A geometric process for spatial reorientation in young children. Nature 370, 57-59. doi: 10.1038/370057a0

Hermer-Vazquez, L., Spelke, E. S., and Katsnelson, A. S. (1999). Sources of flexibility in human cognition: dual-task studies of space and language. Cogn. Psychol. 39, 3-36. doi: 10.1006/cogp.1998. 0713

Jung-Beeman, M. (2005). Bilateral brain processes for comprehending natural language. Trends Cogn. Sci. 9, 512-518. doi: 10.1016/j.tics.2005.09.009

Lupyan, G. (2008). The conceptual grouping effect: categories matter (and named categories matter more). Cognition 108, 566-577. doi: 10.1016/j.cognition.2008.03.009

Lupyan, G. (2009). Extracommunicative functions of language: verbal interference causes selective categorization impairments. Psychon. Bull. Rev. 16, 711-718. doi: 10.3758/PBR.16.4.711

Lupyan, G. (2012a). "What do words do? Toward a theory of language-augmented thought," in The Psychology of Learning and Motivation, Vol. 57, ed B. H. Ross (San Diego, CA: Academic Press), 255-297. doi: 10.1016/B978-0-12-3942937.00007-8

Lupyan, G. (2012b). Linguistically modulated perception and cognition: the label-feedback hypothesis. Front. Cogn. 3:54. doi: 10.3389/fpsyg. 2012.00054

Lupyan, G., and Mirman, D. (2013). Linking language and categorization: evidence from aphasia. Cortex 49, 1187-1194. doi: 10.1016/j.cortex.2012.06.006

Lupyan, G., and Swingley, D. (2012). Self-directed speech affects visual search performance. Q. J. Exp. Psychol. 65, 1068-1085. doi: 10.1080/17470218. 2011.647039

Lupyan, G., Mirman, D., Hamilton, R., and Thompson-Schill, S. L. (2012). Categorization is modulated by transcranial direct current stimulation over left prefrontal cortex. Cognition 124 36-49. doi: 10.1016/j.cognition.2012.04.002

Lupyan, G., Rakison, D. H., and McClelland, J. L. (2007). Language is not just for talking: redundant labels facilitate learning of novel categories Psychol. Sci. 18, 1077-1083. doi: 10.1111/j.14679280.2007.02028.x

MacDonald, M. C., Pearlmutter, N. J., and Seidenberg M. S. (1994). The lexical nature of syntactic ambiguity resolution. Psychol. Rev. 101, 676-703. doi: 10.1037/0033-295X.101.4.676

Newton, A. M., and de Villiers, J. G. (2007). Thinking while talking: adults fail nonverbal falsebelief reasoning. Psychol. Sci. 18, 574-579. doi: 10.1111/j.1467-9280.2007.01942.x

Özgen, E., and Davies, I. R. (2002). Acquisition of categorical color perception: a perceptual learning approach to the linguistic relativity hypothesis. J. Exp. Psychol. Gen. 131, 477-493. doi: 10.1037/0096-3445.131.4.477

Price, C. J. (2000). The anatomy of language: contributions from functional neuroimaging. J. Anat. 197, 335-359. doi: 10.1046/j.1469-7580.2000.1973 0335. $\mathrm{x}$

Roberson, D., Damjanovic, L., and Pilling, M. (2007). Categorical perception of facial expressions: 
evidence for a "category adjustment" model. Mem. Cogn. 35, 1814-1829. doi: 10.3758/BF0319 3512

Ting Siok, W., Kay, P., Wang, W. S. Y., Chan, A. H. D., Chen, L., Luke, K.-K., et al. (2009). Language regions of brain are operative in color perception. Proc. Natl. Acad. Sci. U.S.A. 106, 8140-8145. doi: 10.1073/pnas.0903627106

Twyman, A. D., and Newcombe, N. S. (2010). Five reasons to doubt the existence of a geometric module. Cogn. Sci. 34, 1315-1356. doi: 10.1111/j.15516709.2009.01081.x
Winawer, J., Witthoft, N., Frank, M. C., Wu, L., Wade, A. R., and Boroditsky, L. (2007). Russian blues reveal effects of language on color discrimination. Proc. Natl. Acad. Sci. U.S.A. 104, 7780-7785. doi: 10.1073/pnas.0701644104

Received: 15 July 2013; accepted: 29 August 2013; published online: 17 September 2013.

Citation: Perry LK and Lupyan G (2013) What the online manipulation of linguistic activity can tell us about language and thought. Front. Behav. Neurosci. 7:122. doi: 10.3389/fnbeh.2013.00122
This article was submitted to the journal Frontiers in Behavioral Neuroscience.

Copyright (c) 2013 Perry and Lupyan. This is an open-access article distributed under the terms of the Creative Commons Attribution License (CC BY). The use, distribution or reproduction in other forums is permitted, provided the original author(s) or licensor are credited and that the original publication in this journal is cited, in accordance with accepted academic practice. No use, distribution or reproduction is permitted which does not comply with these terms. 